\title{
Circuit Implementation of Function Cascade Synchronization
}

\author{
Yan-Lin Zhong, ${ }^{1}$ Ming-Chi Lu, ${ }^{1}$ Feng-Wei Tsai, ${ }^{1}$ Chen-An Chan, ${ }^{1}$ Tang-Yi Tsai, ${ }^{2}$ \\ Zai-Xing Zheng, ${ }^{2}$ Bo-Yi Li, ${ }^{2}$ Tsung-Lun Tsai, ${ }^{2}$ Chia-Ju Liu, ${ }^{2}$ and Ming-Chung $\mathrm{Ho}{ }^{*}$ \\ ${ }^{1}$ Department of Physics, National Kaohsiung Normal University, \\ No. 62, Shenjhong Rd., Yanchao District, Kaohsiung City 824, Taiwan \\ ${ }^{2}$ Graduate Institute of Science Education \& Environmental Education, National Kaohsiung Normal University, \\ No. 62, Shenjhong Rd., Yanchao District, Kaohsiung City 824, Taiwan
}

(Received November 30, 2018; accepted June 27, 2019)

Keywords: function cascade synchronization, Lyapunov stability theory, circuit implementation

The Rössler chaotic system was chosen for implementing function cascade synchronization using electronic components. Utilizing the cascade technique with the functional relationship, we achieve the synchronization of specific chaotic system signals. On the basis of the Lyapunov stability theory and by designing the appropriate controllers with the estimated parameters, we successfully make the driving system synchronize with the final response system. Finally, the circuit implementation of function cascade synchronization is in good agreement with numerical and circuit simulations. The circuits inplemented utilizing the function cascade technique could be applied to sensors designed to detect signals of a specific chaotic system.

\section{Introduction}

Chaos is a ubiquitous phenomenon in nonlinear dynamical systems. Its control and synchronization are attractive research topics. There are many powerful methods for controlling and synchronizing chaotic systems, such as the Ott, Grebogi, and Yorke (OGY) methods, ${ }^{(1)}$ adaptive control, ${ }^{(2-4)}$ generalized projective synchronization, ${ }^{(5-7)}$ linear feedback control, ${ }^{(8-10)}$ and active control approach. ${ }^{(11)}$ Several reseachers proposed a cascade synchronization method ${ }^{(12-15)}$ with which the driving system used only one signal to achieve the synchronization of the response system.

The cascade technique has the advantage of high adaptability even when the parameters of the driving system are uncertain for the response system. Additionally, in accordance with the projective synchronization method, the error function with the functional relationship can be designed. Therefore, the cascade synchronization with the functional error function can be used to achieve the synchronization of a specific system successfully. In this study, the Rössler chaotic system is used to achieve the function cascade synchronization. Furthermore, the numerical simulation, circuit simulation, and even circuit implementation are successfully achieved to verify the feasibility of the function cascade synchronization.

*Corresponding author: e-mail: t1603@nknucc.nknu.edu.tw https://doi.org/10.18494/SAM.2019.2217 


\section{Function Cascade Synchronization}

Consider $n$-dimensional chaotic systems following the form

$$
\dot{X}=f(X)+F(X) \Pi, \dot{Y}=g(Y)+G(Y) \tilde{\Pi}+\xi,
$$

where $\boldsymbol{X}$ and $\boldsymbol{Y}$ are the state vectors of the driving and response systems, respectively. Moreover, $\boldsymbol{f}$ and $\boldsymbol{g}$ are continuous vector functions; $\boldsymbol{F}$ and $\boldsymbol{G}$ are state matrix functions; $\boldsymbol{\Pi}$ and $\tilde{\boldsymbol{\Pi}}$ are the theoretical and estimated parameter vectors, respectively; $\xi$ is the controller vector.

The Lyapunov function and its derivative are as follows:

$$
\boldsymbol{V}=\frac{1}{2}\left(\boldsymbol{e}^{\mathrm{T}} \boldsymbol{e}+\breve{\Pi}^{\mathrm{T}} \breve{\Pi}\right), \dot{\boldsymbol{V}}=\boldsymbol{e}^{\mathrm{T}} \dot{\boldsymbol{e}}+\breve{\Pi}^{\mathrm{T}} \dot{\bar{\Pi}},
$$

where $\breve{\Pi}=\tilde{\Pi}-\boldsymbol{\Pi}$. Then, design a suitable error function $\boldsymbol{e}=\boldsymbol{Y}-\boldsymbol{Q} \boldsymbol{X}^{\mathrm{T}}$, where $\boldsymbol{Q}$ is the vector function, and choose the adaptive controller $\xi$ appropriately, and the function cascade synchronization will be achieved if there exists $\dot{\boldsymbol{V}}<\mathbf{0}$ and the form

$$
\lim _{t \rightarrow \infty}\|\boldsymbol{e}\|=\lim _{t \rightarrow \infty}\left\|\boldsymbol{Y}-\boldsymbol{Q} \boldsymbol{X}^{\mathrm{T}}\right\|=0 .
$$

\section{Results}

\subsection{Numerical simulation}

The driving system of the Rössler chaotic system is described as

$$
\left\{\begin{array}{l}
\dot{x}_{d}=-y_{d}-z_{d} \\
\dot{y}_{d}=x_{d}+a y_{d} \\
\dot{z}_{d}=b+z_{d}\left(x_{d}-c\right),
\end{array}\right.
$$

where $a, b$, and $c$ are completely unknown parameters. If $(a, b, c)$ is $(0.2,0.2,5.77)$, the driving system is chaotic (Fig. 1).

The subresponse system is in the form

$$
\left\{\begin{array}{c}
\dot{x}_{r}=-y_{r 1}-z_{d}+\xi_{1} \\
\dot{y}_{r 1}=x_{r}+\alpha_{1} y_{r 1}+\xi_{2} \\
\dot{z}_{d}=b+z_{d}\left(x_{d}-c\right),
\end{array}\right.
$$

and the final response system is in the form 


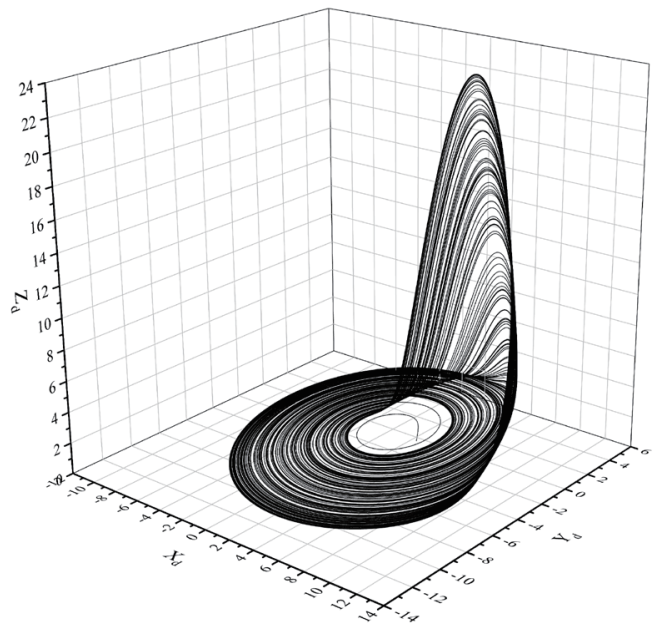

(a)

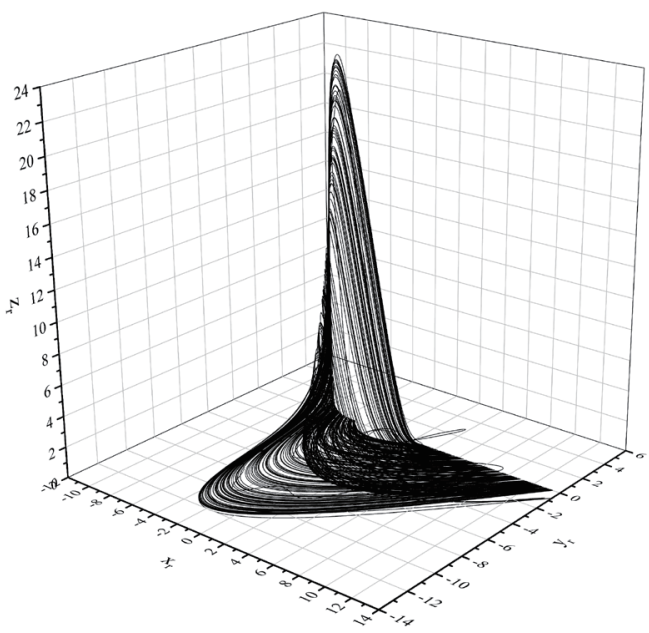

(b)

Fig. 1. 3D plots of Rössler chaotic systems: (a) the driving system with the initial values $(1,1,-1)$ and parameters $(a, b, c)=(0.2,0.2,5.77)$ and $(b)$ the final response system with the initial values $(0.2,0.4,-3)$.

$$
\left\{\begin{array}{l}
\dot{x}_{r}=-y_{r 1}-z_{d}+\xi_{1} \\
\dot{y}_{r}=x_{r}+\alpha_{2} y_{r}+\xi_{3} \\
\dot{z}_{r}=\beta+z_{r}\left(x_{r}-\gamma\right)+\xi_{4},
\end{array}\right.
$$

where $\alpha_{1}$ and $\alpha_{2}$ are the estimated values of the parameter $a ; \beta$ and $\gamma$ are the estimated values of the parameters $b$ and $c$, respectively; $\xi_{1}, \xi_{2}, \xi_{3}$, and $\xi_{4}$ are controllers.

The Lyapunov function and its derivative are as follows:

$$
\begin{aligned}
V & =\frac{1}{2}\left(e_{1}^{2}+e_{2}^{2}+e_{3}^{2}+e_{4}^{2}+\breve{\alpha}_{1}^{2}+\breve{\alpha}_{2}^{2}+\breve{\beta}^{2}+\breve{\gamma}^{2}\right), \\
\dot{V} & =e_{1} \dot{e}_{1}+e_{2} \dot{e}_{2}+e_{3} \dot{e}_{3}+e_{4} \dot{e}_{4}+\breve{\alpha}_{1} \dot{\check{\alpha}}_{1}+\breve{\alpha}_{2} \dot{\dot{\alpha}_{2}}+\breve{\beta} \dot{\ddot{\beta}}+\dot{\gamma} \\
& =e_{1}\left[\left(-y_{r 1}-z_{d}+\xi_{1}\right)-0.1 x_{d}\left(-y_{d}-z_{d}\right)+\left(-y_{d}-z_{d}\right)\right] \\
& +e_{2}\left[\left(x_{r}+\alpha_{1} y_{r 1}+\xi_{2}\right)-\left(x_{d}+a y_{d}\right)\right] \\
& +e_{3}\left[\left(x_{r}+\alpha_{2} y_{r}+\xi_{3}\right)+0.2 y_{d}\left(x_{d}+a y_{d}\right)\right] \\
& +e_{4}\left[\left(\beta+z_{r}\left(x_{r}-\gamma\right)+\xi_{4}\right)-\left(b+z_{d}\left(x_{d}-c\right)\right)\right] \\
& +\dot{\alpha}_{1}\left(\alpha_{1}-a\right)+\dot{\alpha}_{2}\left(\alpha_{2}-a\right)+\dot{\beta}(\beta-b)+\dot{\gamma}(\gamma-c),
\end{aligned}
$$

where $e_{1}=x_{r}-0.05 x_{d}^{2}+x_{d}, e_{2}=y_{r 1}-y_{d}, e_{3}=y_{r}+0.1 y_{d}^{2}, e_{4}=z_{r}-z_{d}, \breve{\alpha}_{1}=\alpha_{1}-a, \breve{\alpha}_{2}=\alpha_{2}-a$, $\breve{\beta}=\beta-b$, and $\breve{\gamma}=\gamma-c$; the controllers and estimated parameters are presented as 


$$
\left\{\begin{array}{l}
\xi_{1}=-e_{1}-\left(-y_{r 1}-z_{d}\right)+0.1 x_{d}\left(-y_{d}-z_{d}\right)-\left(-y_{d}-z_{d}\right) \\
\xi_{2}=-e_{2}-\left(x_{r}+\alpha_{1} y_{r 1}\right)+\left(x_{d}+\alpha_{1} y_{d}\right) \\
\xi_{3}=-e_{3}-\left(x_{r}+\alpha_{2} y_{r}\right)-0.2 y_{d}\left(x_{d}+\alpha_{2} y_{d}\right) \\
\xi_{4}=-e_{4}-\left[\beta+z_{r}\left(x_{r}-\gamma\right)\right]+\left[\beta+z_{d}\left(x_{d}-\gamma\right)\right] \\
\dot{\alpha}_{1}=-e_{2} y_{d} \\
\dot{\alpha}_{2}=-0.2 e_{3} y_{d}^{2} \\
\dot{\beta}=-e_{4} \\
\dot{\gamma}=e_{4} z_{d} .
\end{array}\right.
$$

Substitute Eq. (8) into the derivative of the Lyapunov function Eq. (7); thus, $\dot{V}=-e_{1}^{2}-e_{2}^{2}-e_{3}^{2}-e_{4}^{2}$ and then

$$
\lim _{t \rightarrow \infty}\left\|e_{n}\right\|=0,(n=1,2,3,4)
$$

According to the Lyapunov stability theorem, the method utilizing the function cascade indeed can achieve the synchronization of the Rössler chaotic system with uncertainty parameters, as shown in Fig. 2.

The special feature of the cascade method is that it must proceed through two steps. In each step, one of the signals of the first system remains the same as that of the second system, the error functions are quadratic, and all the parameters are completely unknown, which constitute our study method, that is, function cascade synchronization. Figure 2 shows that the error functions tend to be zero when the controller are input to the subresponse and final response systems, which means that the function cascade synchronization is achieved.

\subsection{Circuit simulation and circuit implementation}

The circuit design of the cascade synchronization of the Rössler chaotic system is shown in Fig. 3, in which LF353 and AD633 are selected as the amplifiers and multipliers, respectively, and the capacitors are all $0.1 \mu \mathrm{F}$.

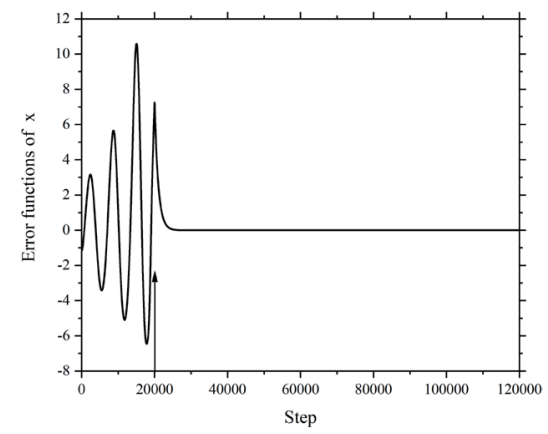

(a)

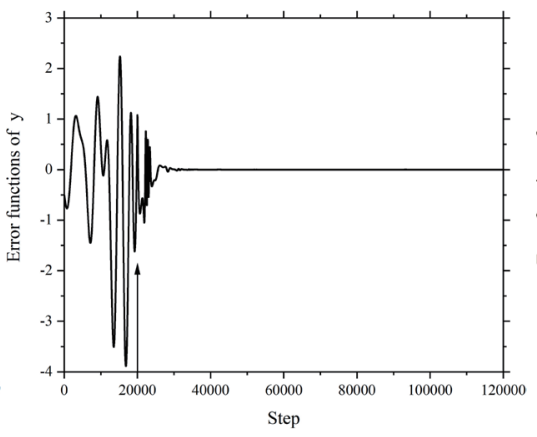

(b)

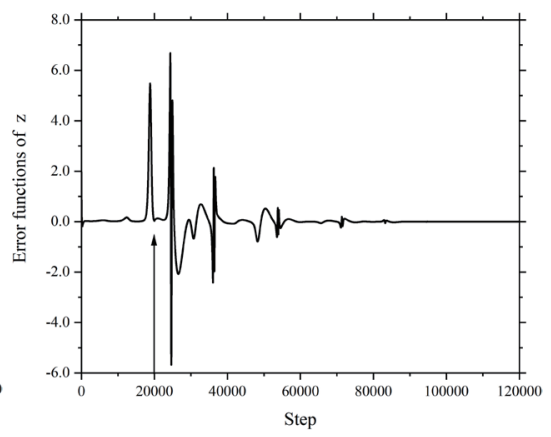

(c)

Fig. 2. Numerical simulation plots of error functions: (a) $x_{r}-0.05 x_{d}^{2}+x_{d}$, (b) $y_{r}+0.1 y_{d}^{2}$, and (c) $z_{r}-z_{d}$. The arrow indicates the time at which function cascade synchronization starts. 

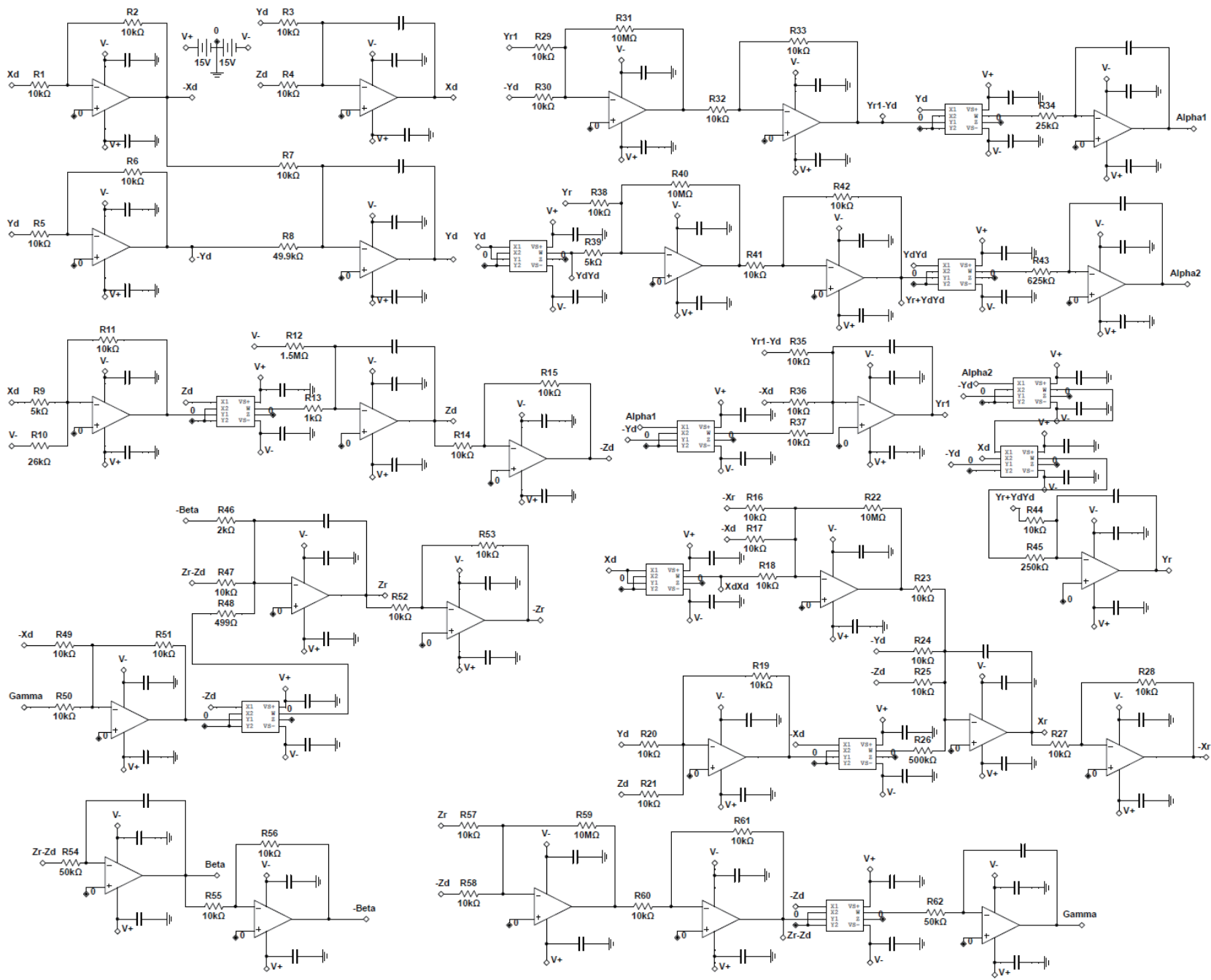

Fig. 3. Circuit design of the cascade synchronization of the Rössler chaotic system.

Considering the operating range of electronic components, the system output voltage is less than $\pm 13.5 \mathrm{~V}$, such that the design of the circuit must utilize the scaling factors. The scaling factors of the circuit are $x_{d}=2 X_{d}, y_{d}=2 Y_{d}, z_{d}=2 Z_{d}, x_{r}=2 X_{r}, y_{r 1}=2 Y_{r 1}, z_{r}=2 Z_{r}, \alpha_{1}=10 A_{1}, \alpha_{2}$ $=10 A_{2}, \beta=2 B$, and $\gamma=2 \Gamma$. The driving system parameters $a, b$, and $c$ are $0.2,0.2$, and 5.77, respectively.

Then, transform the time scale $\tau=1000 t$, and the driving system (4) becomes

$$
\left\{\begin{array}{l}
\dot{X}_{d}=-1000\left(Y_{d}+Z_{d}\right) \\
\dot{Y}_{d}=1000\left(X_{d}+0.2 Y_{d}\right) \\
\dot{Z}_{d}=100+1000 Z_{d}\left(2 X_{d}-5.77\right)
\end{array}\right.
$$


the subresponse system (5) becomes

$$
\left\{\begin{array}{l}
\dot{X}_{r}=-1000\left(X_{r}-0.1 X_{d} X_{d}+X_{d}\right)+100\left(2 X_{d}-10\right)\left(-Y_{d}-Z_{d}\right) \\
\dot{Y}_{r 1}=-1000\left(Y_{r 1}-Y_{d}\right)+1000\left(X_{d}+10 A_{1} Y_{d}\right) \\
\dot{Z}_{d}=100+1000 Z_{d}\left(2 X_{d}-5.77\right)
\end{array}\right.
$$

the final response system (6) becomes

$$
\left\{\begin{array}{l}
\dot{X}_{r}=-1000\left(X_{r}-0.1 X_{d} X_{d}+X_{d}\right)+100\left(2 X_{d}-10\right)\left(-Y_{d}-Z_{d}\right) \\
\dot{Y}_{r}=-1000\left(Y_{r}+0.2 Y_{d} Y_{d}\right)-400 Y_{d}\left(X_{d}+10 A_{2} Y_{d}\right) \\
\dot{Z}_{r}=-1000\left(Z_{r}-Z_{d}\right)+1000\left(B+2 Z_{d}\left(X_{d}-\Gamma\right)\right)
\end{array}\right.
$$

and the estimated parameters (8) become

$$
\left\{\begin{aligned}
\dot{A}_{1} & =-400 Y_{d}\left(Y_{r 1}-Y_{d}\right) \\
\dot{A}_{2} & =-800 Y_{d} Y_{d}\left(Y_{r}+0.2 Y_{d} Y_{d}\right) \\
\dot{B} & =-1000\left(Z_{r}-Z_{d}\right) \\
\dot{\Gamma} & =2000 Z_{d}\left(Z_{r}-Z_{d}\right) .
\end{aligned}\right.
$$

To facilitate the circuit design, the driving system (10) is rewritten as

$$
\left\{\begin{array}{l}
\dot{X}_{d}=-\frac{1}{C}\left(\frac{Y_{d}}{R_{3}}+\frac{Z_{d}}{R_{4}}\right) \\
\dot{Y}_{d}=-\frac{1}{C}\left(-\frac{X_{d}}{R_{7}}-\frac{Y_{d}}{R_{8}}\right) \\
\dot{Z}_{d}=-\frac{1}{C}\left(\frac{V_{-}}{R_{12}}-\frac{Z_{d}\left(X_{d}+V_{-}\right)}{10 R_{13}}\right)
\end{array}\right.
$$

the subresponse system (11) is rewritten as

$$
\left\{\begin{array}{l}
\dot{X}_{r}=-\frac{1}{C}\left(\frac{\left(X_{r}-0.1 X_{d} X_{d}+X_{d}\right)}{R_{23}}+\frac{X_{d}\left(Y_{d}+Z_{d}\right)}{10 R_{26}}-\frac{Y_{d}}{R_{24}}-\frac{Z_{d}}{R_{25}}\right) \\
\dot{Y}_{r 1}=-\frac{1}{C}\left(\frac{Y_{r 1}-Y_{d}}{R_{35}}-\frac{X_{d}}{R_{36}}-\frac{A_{1} Y_{d}}{10 R_{37}}\right) \\
\dot{Z}_{d}=-\frac{1}{C}\left(\frac{V_{-}}{R_{12}}-\frac{Z_{d}\left(X_{d}+V_{-}\right)}{10 R_{13}}\right),
\end{array}\right.
$$


the final response system (12) is rewritten as

$$
\left\{\begin{array}{l}
\dot{X}_{r}=-\frac{1}{C}\left(\frac{\left(X_{r}-0.1 X_{d} X_{d}+X_{d}\right)}{R_{23}}+\frac{X_{d}\left(Y_{d}+Z_{d}\right)}{10 R_{26}}-\frac{Y_{d}}{R_{24}}-\frac{Z_{d}}{R_{25}}\right) \\
\dot{Y}_{r}=-\frac{1}{C}\left(\frac{Y_{r}+0.1 Y_{d} Y_{d}}{R_{44}}+\frac{Y_{d}\left(X_{d}+0.1 A_{1} Y_{d}\right)}{10 R_{45}}\right) \\
\dot{Z}_{r}=-\frac{1}{C}\left(\frac{Z_{r}-Z_{d}}{R_{47}}-\frac{B}{R_{46}}-\frac{Z_{d}\left(X_{d}-\Gamma\right)}{10 R_{48}}\right),
\end{array}\right.
$$

and estimated parameters (13) are rewritten as

$$
\left\{\begin{array}{l}
\dot{A}_{1}=-\frac{Y_{d}}{10 C}\left(\frac{Y_{r 1}-Y_{d}}{R_{34}}\right) \\
\dot{A}_{2}=-\frac{0.1 Y_{d} Y_{d}}{10 C}\left(\frac{Y_{r}+0.1 Y_{d} Y_{d}}{R_{43}}\right) \\
\dot{B}=-\frac{1}{C}\left(\frac{Z_{r}-Z_{d}}{R_{54}}\right) \\
\dot{\Gamma}=-\frac{1}{C}\left(\frac{-Z_{d}\left(Z_{r}-Z_{d}\right)}{10 R_{62}}\right) .
\end{array}\right.
$$

The driving system output $\left(x_{d}, y_{d}, z_{d}\right)$ is decreased by half and then the resistors $R_{d}=10 \mathrm{k} \Omega$ ( $d=1-7,11,14$, and 15$), R_{8}=49.9 \mathrm{k} \Omega, R_{9}=5 \mathrm{k} \Omega, R_{10}=26 \mathrm{k} \Omega, R_{12}=1.5 \mathrm{M} \Omega$, and $R_{13}=1 \mathrm{k} \Omega$. Then, the subresponse system output $\left(x_{r}, y_{r 1}, z_{d}\right)$ is decreased by half, in which the $z_{d}$ signal is identical to the driving system signal, and the resistors $R_{r 1}=10 \mathrm{k} \Omega\left(r_{1}=16-21,23-25,27-30\right.$, 32,33 , and 35-37), $R_{22}=R_{31}=10 \mathrm{M} \Omega, R_{26}=500 \mathrm{k} \Omega$, and $R_{34}=25 \mathrm{k} \Omega$. Additionally, the final system output $\left(y_{r}, z_{r}\right)$ is decreased by half, in which the $x_{r}$ signal is identical to the subresponse system signal, and the resistors $R_{r}=10 \mathrm{k} \Omega(r=38,41,42,44,47,49-53,55-58,60$, and 61), $R_{39}=5 \mathrm{k} \Omega, R_{40}=R_{59}=10 \mathrm{M} \Omega, R_{43}=625 \mathrm{k} \Omega, R_{45}=250 \mathrm{k} \Omega, R_{46}=2 \mathrm{k} \Omega, R_{48}=499 \Omega$, and $R_{54}=R_{62}=50 \mathrm{k} \Omega$.

Owing to the characteristic of the Rössler chaotic system, the circuit does not interfere with any noise and it is possible to implement the synchronization circuit. Therefore, the supply voltages of all the amplifiers in the circuit design are connected to an additional capacitor of $0.1 \mu \mathrm{F}$ and then grounded. In this way, the signals in the circuit can be pure.

Finally, the phase diagram obtained by circuit simulation (Fig. 4) and that obtained by circuit implementation (Fig. 5) show diagonal lines, which means that two variables are identical, that is, the function of the driving system and the final response system successfully achieve the function cascade synchronization. 


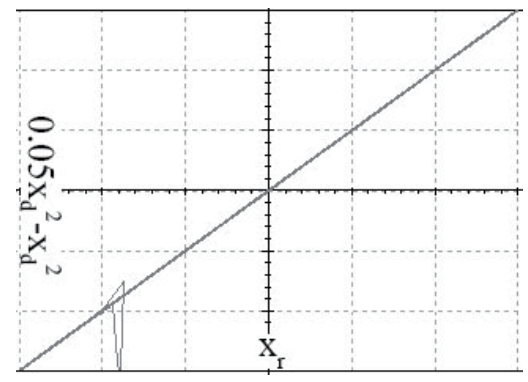

(a)

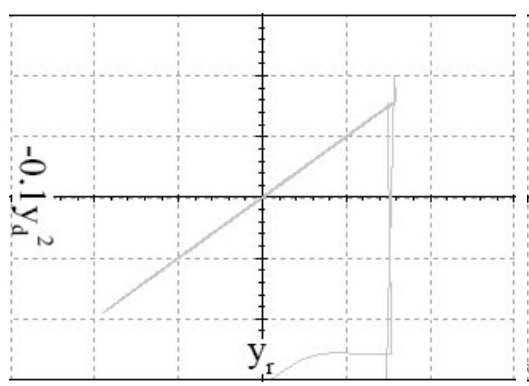

(b)

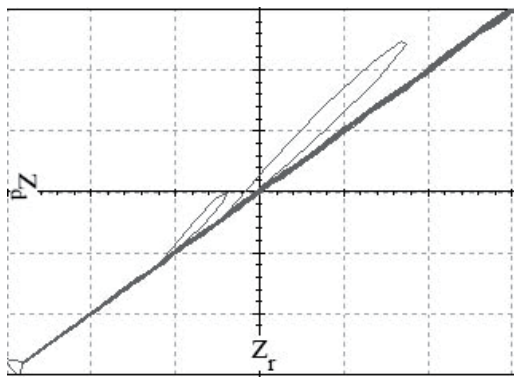

(c)

Fig. 4. Phase diagrams of circuit simulation: (a) $x_{r}$ vs $0.05 x_{d}^{2}-x_{d}$, (b) $y_{r}$ vs $-0.1 y_{d}^{2}$, and (c) $z_{r}$ vs $z_{d}$.

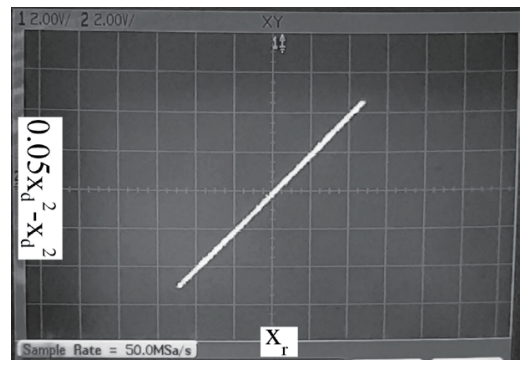

(a)

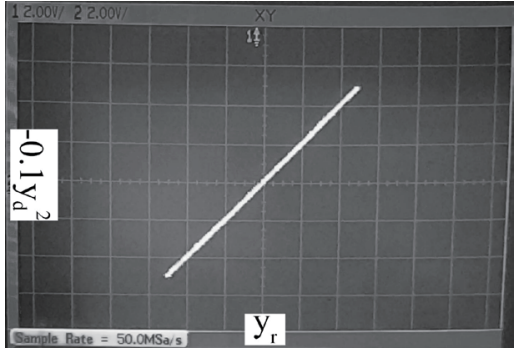

(b)

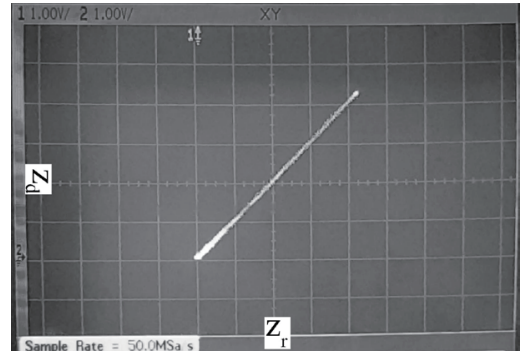

(c)

Fig. 5. Phase diagrams of circuit implementation: (a) $x_{r}$ vs $0.05 x_{d}^{2}-x_{d}$, (b) $y_{r}$ vs $-0.1 y_{d}^{2}$, and (c) $z_{r}$ vs $z_{d}$.

\section{Conclusions}

Firstly, the result of the numerical simulation shows the feasibility and effectiveness of the function cascade method. Secondly, despite the specific characteristic of the Rössler chaotic system, the complexity of the function cascade method, and even the difficulty in real circuit implementation, we overcome all the problems encountered and achieve the circuit implementation of the function cascade synchronization of the Rössler chaotic system by designing the circuit skillfully, that is, connecting capacitors and grounding to avoid any noise interference. Finally, the result of the circuit implementation verifies the success of our scheme for implementing the circuit of the function cascade synchronization of the Rössler chaotic system with fully unknown parameters.

\section{References}

1 E. Ott, C. Grebogi, and J. A. Yorke: Phys. Rev. Lett. 64 (1990) 23. https://doi.org/10.1103/PhysRevLett.64.1196

2 Y. Wang, Z. H. Guan, and H. O. Wang: Phys. Lett. A 312 (2003) 1. https://doi.org/10.1016/S03759601(03)00573-5

3 M. T. Yassen: Appl. Math. Comput. 135 (2003) 1. https://doi.org/10.1016/S0096-3003(01)00318-6

4 J. Lu, X. Wu, X. Han, and J. Lü: Phys. Lett. A 329 (2004) 4. https://doi.org/10.1016/j.physleta.2004.07.024 
5 R. Mainieri and J. Rehacek: Phys. Rev. Lett. 82 (1999) 15. https://doi.org/10.1103/PhysRevLett.82.3042

6 G. Wen and D. Xu: Chaos, Solitons Fractals 26 (2005) 1. https://doi.org/10.1016/j.chaos.2004.09.117

7 J. Yan and C. Li: Chaos, Solitons Fractals 26 (2005) 4. https://doi.org/10.1016/j.chaos.2005.02.034

8 C. C. Hwang, J. Y. Hsieh, and R. S. Lin: Chaos, Solitons Fractals 8 (1997) 9. https://doi.org/10.1016/S09600779(96)00150-6

9 X. Zeng, J. Ruan, and L. Li: Commun. Nonlinear Sci. Numer. Simul. 4 (1999) 2. https://doi.org/10.1016/S10075704(99)90032-1

10 J. Lü and J. Lu: Chaos, Solitons Fractals 17 (2003) 1. https://doi.org/10.1016/S0960-0779(02)00456-3

11 H. N. Agiza and M.T. Yassen: Phys. Lett. A 278 (2001) 4. https://doi.org/10.1016/S0375-9601(00)00777-5

12 T. L. Carroll and L.M. Pecora: Physica D 67 (1993) 1. https://doi.org/10.1016/0167-2789(93)90201-B

13 Y. Chen, H. An, and Z. Li: Appl. Math. Comput. 197 (2008) 1. https://doi.org/10.1016/j.amc.2007.07.036

14 H. An and Y. Chen: Commun. Nonlinear Sci. Numer. Simul. 13 (2008) 10. https://doi.org/10.1016/ j.cnsns.2007.05.029

15 M. C. Lu, C. J. Liu, M. C. Ho, H. Jan, and C. Y. Chou: Chin. J. Phys. 48 (2010) 4. 